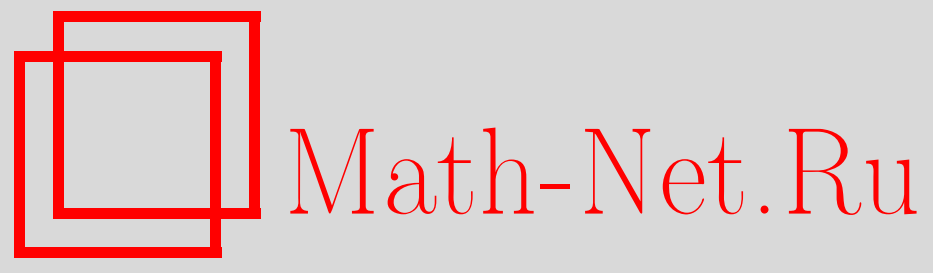

О. А. Репин, А. В. Тарасенко, Нелокальная задача для уравнения с частной производной дробного порядка, Вестн. Сам. гос. техн. ун-та. Сер. Физ.-мат. науки, 2015, номер 1, 78-86

DOI: https://doi.org/10.14498/vsgtu1398

Использование Общероссийского математического портала MathNet.Ru подразумевает, что вы прочитали и согласны с пользовательским соглашением

http://www. mathnet.ru/rus/agreement

Параметры загрузки:

IP: 54.198 .67 .100

26 апреля 2023 г., 15:55:34

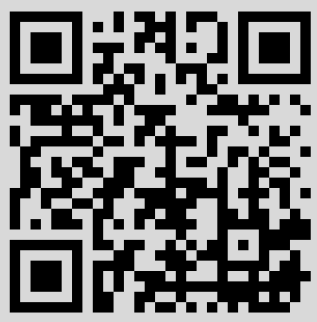


УДК 517.956.6

\title{
НЕЛОКАЛЬНАЯ ЗАДАЧА ДЛЯ УРАВНЕНИЯ С ЧАСТНОЙ ПРОИЗВОДНОЙ ДРОБНОГО ПОРЯДКА
}

\author{
O. A. Репин ${ }^{1,2}$, A. В. Тарасенко ${ }^{3}$ \\ 1 Самарский государственный экономический университет, \\ Россия, 443090, Самара, ул. Советской Армии, 141. \\ 2 Самарский государственный технический университет, \\ Россия, 443100, Самара, ул. Молодогвардейская, 244. \\ 3 Самарский государственный архитектурно-строительный университет, \\ Россия, 443001, Самара, ул. Молодогвардейская, 194.
}

\section{Аннотация}

Для уравнения с частными производными смешанного типа (уравнение диффузии дробного порядка) в конечной области исследована нелокальная задача, краевое условие которой содержит линейную комбинацию обобщённых операторов дробного интегро-дифференцирования от значений решения на характеристиках со значениями решения и его производной на линии вырождения. Единственность решения задачи доказана с помощью модифицированного метода Трикоми, а существование решения эквивалентно редуцировано к вопросу разрешимости интегральных уравнений Фредгольма второго рода.

Ключевые слова: оператор дробного интегро-дифференцирования, краевая задача, интегральное уравнение Фредгольма второго рода.

doi: http://dx.doi.org/10.14498/vsgtu1398

\section{1. Постановка задачи. Рассмотрим уравнение}

$$
\begin{cases}u_{x x}-D_{0+, y}^{\alpha} u=0 & (y>0,0<\alpha<1) \\ y^{2 m} u_{x x}+y u_{y y}+\lambda u_{y}=0 & (y<0)\end{cases}
$$

где $D_{0+, y}^{\alpha}-$ частная дробная производная Римана-Лиувилля порядка $\alpha$ от функции $u(x, y)$ по второй переменной [1, с. 341]:

$$
\left(D_{0+, y}^{\alpha} u\right)(x, y)=\frac{\partial}{\partial y} \frac{1}{\Gamma(1-\alpha)} \int_{0}^{y} \frac{u(x, t) d t}{(y-t)^{\alpha}} \quad(0<\alpha<1, y>0) \text {, }
$$

(C) 2015 Самарский государственный технический университет.

\section{Образец для цитирования}

Р еп и н О. А., Т ар а с ен к о А. В. Нелокальная задача для уравнения с частной производной дробного порядка // Вестн. Сам. гос. техн. ун-та. Сер. Физ.-мат. науки, 2015. Т. 19, № 1. С. 78-86. doi: 10.14498/vsgtu1398.

\section{Сведения об авторах}

Олег Александрович Репин (д.ф.-м.н., проф.; matstat@mail.ru; автор, ведущий переписку), заведующий кафедрой, каф. математической статистики и эконометрики ${ }^{1}$ професcop, каф. прикладной математики и информатики ${ }^{2}$.

Анна Валеръевна Тарасенко (к.ф.-м.н., доц.; tarasenko.a.v@mail.ru), доцент, каф. высшей математики. 
$m$ - натуральное число, $\lambda=$ const, $(1-2 m) / 2 \leqslant \lambda<1$ в конечной области $\Omega$, ограниченной отрезками $A A_{0}, B B_{0}, A_{0} B_{0}$ прямых $x=0, x=1, y=1$ соответственно и характеристиками уравнения (1) при $y<0$ :

$$
A C: x-\frac{2}{2 m+1}(-y)^{\frac{2 m+1}{2}}=0, \quad B C: x+\frac{2}{2 m+1}(-y)^{\frac{2 m+1}{2}}=1
$$

Пусть $\Omega^{+}=\Omega \cap(y>0), \Omega^{-}=\Omega \cap(y<0), I \equiv A B-$ единичный интервал $0<x<1$ прямой $y=0$.

ЗАДАчА.Найти решение $u(x, y)$ уравнения (1) в области $\Omega$, удовлетворяющее краевым условиям

$$
u(0, y)=\varphi_{1}(y), \quad u(1, y)=\varphi_{2}(y), \quad 0<y<1
$$

$$
\begin{aligned}
& a(x)\left(I_{0+}^{\alpha_{1}, \beta_{1}, \eta_{1}} \delta(t)\right.\left.u\left[\Theta_{0}(t)\right]\right)(x)+b(x)\left(I_{1-}^{\alpha_{2}, \beta_{2}, \eta_{2}} w(t) u\left[\Theta_{1}(t)\right]\right)(x)+ \\
&+c(x) u(x, 0)+d(x) \lim _{y \rightarrow 0-0}(-y)^{\lambda} u_{y}(x, y)=\gamma(x), \quad x \in I
\end{aligned}
$$

и условиям сопряжения

$$
\begin{gathered}
\lim _{y \rightarrow 0+0}\left(y^{1-\alpha} u(x, y)\right)=\lim _{y \rightarrow 0-0} u(x, y), \quad x \in \bar{I}, \\
\lim _{y \rightarrow 0+0}\left[y^{1-\alpha}\left(y^{1-\alpha} u(x, y)\right)_{y}\right]=\lim _{y \rightarrow 0-0}(-y)^{\lambda} u_{y}(x, y), \quad x \in I .
\end{gathered}
$$

Здесъ $\varphi_{i}(y)(i=1,2), a(x), b(x), c(x), d(x), \gamma(x), \delta(x), w(x)-$ заданные функиии, причем

$$
\begin{gathered}
a^{2}(x)+b^{2}(x)+c^{2}(x)+d^{2}(x) \neq 0, \quad b(0)=0, \\
a(x), b(x), c(x), d(x), \gamma(x) \in C^{1}(\bar{I}) \cap C^{3}(I), \\
y^{1-\alpha} \varphi_{1}(y), y^{1-\alpha} \varphi_{2}(y) \in C(\bar{I}), \quad \varphi_{1}(0)=\varphi_{2}(0)=0 ;
\end{gathered}
$$

$\Theta_{0}(x)$ и $\Theta_{1}(x)$ - точки пересечения характеристик уравнения (1), выходящих из точки $(x, 0) \in I$, с характеристиками $A C$ и $B C$ соответственно; $\left(I_{0+}^{\alpha, \beta, \eta} f\right)(x),\left(I_{1-}^{\alpha, \beta, \eta} f\right)(x)$ - обобщённые операторы дробного интегро-дифберениирования с гипергеометрической функиией Гаусса $F(a, b ; c ; z)$, введённье в работе [2] (см. также [1, с. 326, 327] [3, с. 14]) и имеющие при действительных $\alpha, \beta, \eta и x>0$ следуюший вид:

$$
\left(I_{0+}^{\alpha, \beta, \eta} f\right)(x)=\left\{\begin{array}{l}
\frac{x^{-\alpha-\beta}}{\Gamma(\alpha)} \int_{0}^{x}(x-t)^{\alpha-1} F\left(\alpha+\beta,-\eta ; \alpha ; 1-\frac{t}{x}\right) f(t) d t \\
\left(\frac{d}{d x}\right)^{n}\left(I_{0+}^{\alpha+n, \beta-n, \eta-n} f(t)\right)(x) \\
\quad(\alpha \leqslant 0, n=[-\alpha]+1)
\end{array}\right.
$$




$$
\left(I_{1-}^{\alpha, \beta, \eta} f\right)(x)=\left\{\begin{array}{c}
\frac{(1-x)^{-\alpha-\beta}}{\Gamma(\alpha)} \int_{x}^{1}(t-x)^{\alpha-1} F\left(\alpha+\beta,-\eta ; \alpha ; \frac{t-x}{1-x}\right) f(t) d t \\
\left(-\frac{d}{d x}\right)^{n}\left(I_{1-}^{\alpha+n, \beta-n, \eta-n} f(t)\right)(x) \\
(\alpha \leqslant 0), n=[-\alpha]+1),
\end{array}\right.
$$

в частности

$$
\left(I_{0+}^{0,0, \eta} f\right)(x)=f(x), \quad\left(I_{1-}^{0,0, \eta} f\right)(x)=f(x) .
$$

А. Н. Кочубей [4] назвал уравнение (1) при $y>0$ уравнением диффузии дробного порядка.

При $y<0$ уравнение $(1)$ является моделью гиперболических уравнений второго порядка, тип и порядок которых вырождаются на одном и том же $(n-1)$-мерном континууме [5, с. 274].

Будем искать решение поставленной задачи в классе дважды дифференцируемых в области $\Omega$ функций $u(x, y)$, таких, что

$$
\begin{gathered}
y^{1-\alpha} u(x, y) \in C\left(\overline{\Omega^{+}}\right), \quad u(x, y) \in C\left(\overline{\Omega^{-}}\right), \\
y^{1-\alpha}\left(y^{1-\alpha} u_{y}\right)_{y} \in C\left(\Omega^{+} \cup\{(x, y): 0<x<1, y=0\}\right), \\
u_{x x} \in C\left(\Omega^{+} \cup \Omega^{-}\right), \quad u_{y y} \in C\left(\Omega^{-}\right) .
\end{gathered}
$$

2. Единственность решения задачи. Пусть существует решение исследуемой задачи. Введём следующие обозначения:

$$
\begin{gathered}
\lim _{y \rightarrow 0+0} y^{1-\alpha} u(x, y)=\tau_{1}(x), \quad \lim _{y \rightarrow 0-0} u(x, y)=\tau_{2}(x), \\
\lim _{y \rightarrow 0+0} y^{1-\alpha}\left(y^{1-\alpha} u(x, y)\right)_{y}=\nu_{1}(x), \quad \lim _{y \rightarrow 0-0}(-y)^{\lambda} u_{y}(x, y)=\nu_{2}(x), \\
\tau_{1}(x)=\tau_{2}(x)=\tau(x), \quad \nu_{1}(x)=\nu_{2}(x)=\nu(x) .
\end{gathered}
$$

Теорема 1.В области $\Omega$ не может существовать более одного решения задачи (1)-(5), если $0<\alpha<1,(1-2 m) / 2<\lambda<1$ и если

$$
\alpha_{1}=\alpha_{2}=\beta-1
$$

и либо

$$
\beta_{1}=\beta_{2}=1-2 \beta, \quad \eta_{1}=\eta_{2}=0, \quad \delta(x)=w(x)=1,
$$

либо

$$
\beta_{1}=\beta_{2}=0, \quad \eta_{1}=\eta_{2}=1-2 \beta, \quad \delta(x)=x^{2 \beta-1}, \quad w(x)=(1-x)^{2 \beta-1},
$$

и выполнении условиц

$$
\mu(x)=k_{0} a(x)+b(x)-\frac{\Gamma(\beta)}{\Gamma(2 \beta)} d(x) \neq 0 \quad \forall x \in \bar{I}, \quad k_{0}=-\frac{k_{2}}{k_{1}},
$$

$$
\frac{a(1)}{\mu(1)} \geqslant 0, \quad \frac{b(0)}{\mu(0)} \leqslant 0, \quad\left[\frac{a(x)}{\mu(x)}\right]^{\prime} \leqslant 0, \quad\left[\frac{b(x)}{\mu(x)}\right]^{\prime} \geqslant 0, \quad \frac{c(x)}{\mu(x)} \geqslant 0 \quad \forall x \in \bar{I}
$$


uภu

$$
\alpha_{1}=\alpha_{2}=-\beta
$$

и либо

$$
\beta_{1}=\beta_{2}=0, \quad \eta_{1}=\eta_{2}=2 \beta-1, \quad \delta(x)=w(x)=1,
$$

либо

$$
\beta_{1}=\beta_{2}=2 \beta-1, \quad \eta_{1}=\eta_{2}=0, \quad \delta(x)=x^{2 \beta-1}, \quad w(x)=(1-x)^{2 \beta-1},
$$

и выполнении условий

$$
\begin{gathered}
E(x)=k_{1}(a(x)+b(x))+c(x) \neq 0 \quad \forall x \in \bar{I}, \\
\frac{a(1)}{E(1)}+\frac{b(0)}{E(0)} \geqslant 0, \quad\left[\frac{a(x)}{E(x)}\right]^{\prime} \leqslant 0, \quad\left[\frac{b(x)}{E(x)}\right]^{\prime} \geqslant 0, \quad \frac{d(x)}{E(x)} \geqslant 0 \quad \forall x \in \bar{I},
\end{gathered}
$$

$2 \partial e$

$$
k_{1}=\frac{\Gamma(2 \beta)}{\Gamma(\beta)}, \quad k_{2}=-\frac{\Gamma(1-2 \beta)}{2 \Gamma(1-\beta)}\left(\frac{2 m+1}{4}\right), \quad \beta=\frac{2 m-1+2 \lambda}{2(2 m+1)}, \quad 0<\beta<\frac{1}{2} .
$$

Доказательств о. Известно [6, 7], что функциональное соотношение между $\tau(x)$ и $\nu(x)$, принесённое из параболической области $\Omega^{+}$на линию $y=0$, имеет вид

$$
\nu(x)=\frac{1}{\Gamma(1+\alpha)} \tau^{\prime \prime}(x) .
$$

Рассмотрим интеграл

$$
I^{*}=\int_{0}^{1} \tau(x) \nu(x) d x .
$$

Подставим $\nu(x)$ из $(16)$ в $I^{*}$. Учитывая, что $\tau(0)=\varphi_{1}(0)=\tau(1)=\varphi_{2}(0)=0$, получим

$$
I^{*}=-\frac{1}{\Gamma(1+\alpha)} \int_{0}^{1}\left[\tau^{\prime}(x)\right]^{2} d x \leqslant 0 .
$$

Далее на основании результатов работы [8] выпишем функциональное соотношение между $\tau(x)$ и $\nu(x)$, принесённое на $\bar{I}$ из гиперболической части $\Omega^{-}$смешанной области $\Omega$ в двух случаях.

Первый случай. Пусть выполняются условия (6)-(10) теоремы 1. Тогда

$$
\nu(x)=A_{1}(x)\left(D_{0+}^{1-2 \beta} \tau\right)(x)+B_{1}(x)\left(D_{1-}^{1-2 \beta} \tau\right)(x)+C_{1}(x) \tau(x)-\frac{\Gamma(\beta)}{\Gamma(2 \beta) \mu(x)} \gamma(x),
$$

где $\left(D_{0+}^{1-2 \beta} f\right)(x)$ и $\left(D_{1-}^{1-2 \beta} f\right)(x)$ - операторы дробного дифференцирования Римана-Лиувилля [1, с. 44];

$$
A_{1}(x)=\frac{a(x)}{\mu(x)}, \quad B_{1}(x)=\frac{b(x)}{\mu(x)}, \quad C_{1}(x)=\frac{\Gamma(\beta) c(x)}{\Gamma(2 \beta) \mu(x)} .
$$


Второй случай. Пусть выполняются условия (11)-(15) теоремы 1. Тогда

$$
\tau(x)=a_{1}(x)\left(I_{0+}^{1-2 \beta} \nu\right)(x)+b_{1}(x)\left(I_{1-}^{1-2 \beta} \nu\right)(x)+c_{1}(x) \nu(x)+\gamma_{1}(x),
$$

где $\left(I_{0+}^{1-2 \beta} f\right)(x)$ и $\left(I_{1-}^{1-2 \beta} f\right)(x)$ - дробные интегралы Римана-Лиувилля [1, c. 42];

$$
a_{1}(x)=-k_{2} \frac{a(x)}{E(x)}, \quad b_{1}(x)=-k_{2} \frac{b(x)}{E(x)}, \quad c_{1}(x)=\frac{d(x)}{E(x)}, \quad \gamma_{1}=\frac{\gamma(x)}{k_{1} E(x)} .
$$

В работе [8] с учетом функционального соотношения (18) или (19) доказано, что $I^{*} \geqslant 0$. А тогда, учитывая (17), имеем $I^{*}=0$.

Далее схема доказательства тождества $u(x, y) \equiv 0$ аналогична $[8,9]$.

Теорема 2.В области $\Omega$ не может существовать более одного решения задачи (1)-(5), если $0<\alpha<1, \lambda=(1-2 m) / 2(\beta=0)$,

$$
\begin{aligned}
& E_{1}(x)=2 d(x)-a(x)-b(x) \neq 0 \quad \forall x \in \bar{I} \\
& \left(\frac{b(x)-a(x)}{E_{1}(x)}\right)^{\prime} \leqslant 0, \quad \frac{c(x)}{E_{1}(x)} \leqslant 0 \quad \forall x \in \bar{I} .
\end{aligned}
$$

Доказательство теоремы 2 непосредственно следует из равенства $I^{*}=0$ с учётом соотношения [10]

$$
\nu(x)=A_{2}(x) \tau^{\prime}(x)+B_{2}(x) \tau(x)+\gamma_{2}(x),
$$

где

$$
A_{2}(x)=\frac{b(x)-a(x)}{E_{1}(x)}, \quad B_{2}(x)=-\frac{2 c(x)}{E_{1}(x)}, \quad \gamma_{2}(x)=\frac{2 \gamma(x)}{E_{1}(x)} .
$$

3. Существование решения задачи. Интегрируя (16) дважды от 0 до $x$ и учитывая условия (2), получим

$$
\tau(x)=\Gamma(1+\alpha)\left[\int_{0}^{x}(x-\xi) \nu(\xi) d \xi-x \int_{0}^{1}(1-\xi) \nu(\xi) d \xi\right] .
$$

Рассмотрим вначале случай $(1-2 m) / 2<\lambda<1$. Исключив $\nu(x)$ из (18) и (21), будем иметь

$$
\begin{aligned}
\tau(x)+ & \frac{\Gamma(1+\alpha)}{\Gamma(2 \beta)}\left\{x \int _ { 0 } ^ { 1 } ( 1 - \xi ) \left[A_{1}(\xi) \frac{d}{d \xi} \int_{0}^{\xi} \frac{\tau(t) d t}{(\xi-t)^{1-2 \beta}}-\right.\right. \\
-B_{1}(\xi) & \left.\left.\frac{d}{d \xi} \int_{\xi}^{1} \frac{\tau(t) d t}{(t-\xi)^{1-2 \beta}}+\Gamma(2 \beta) C_{1}(\xi) \tau(\xi)-\frac{\Gamma(\beta) \gamma(\xi)}{\mu(\xi)}\right] d \xi\right\}- \\
& -\frac{\Gamma(1+\alpha)}{\Gamma(2 \beta)}\left\{\int _ { 0 } ^ { x } ( x - \xi ) \left[A_{1}(\xi) \frac{d}{d \xi} \int_{0}^{\xi} \frac{\tau(t) d t}{(\xi-t)^{1-2 \beta}}-\right.\right. \\
- & \left.\left.B_{1}(\xi) \frac{d}{d \xi} \int_{\xi}^{1} \frac{\tau(t) d t}{(t-\xi)^{1-2 \beta}}+\Gamma(2 \beta) C_{1}(\xi) \tau(\xi)-\frac{\Gamma(\beta) \gamma(\xi)}{\mu(\xi)}\right] d \xi\right\} .
\end{aligned}
$$


Проинтегрировав в полученном выражении двойные интегралы по частям, а затем поменяв порядок интегрирования, получим

$$
\tau(x)+\int_{0}^{1} K(x, t) \tau(t) d t=f(x)
$$

где

$$
K(x, t)= \begin{cases}K_{1}(x, t), & t \leqslant x, \\ K_{2}(x, t), & t \geqslant x,\end{cases}
$$

$$
\begin{aligned}
& K_{1}(x, t)=-\frac{\Gamma(1+\alpha)}{\Gamma(2 \beta)} x\left[\int_{t}^{x} \frac{\left[(1-\xi) A_{1}(\xi)\right]^{\prime} d \xi}{(\xi-t)^{1-2 \beta}}+\right. \\
&\left.+\int_{x}^{1} \frac{\left[(1-\xi) B_{1}(\xi)\right]^{\prime} d \xi}{(\xi-t)^{1-2 \beta}}+\int_{0}^{t} \frac{\left[(1-\xi) B_{1}(\xi)\right]^{\prime} d \xi}{(t-\xi)^{1-2 \beta}}\right]+ \\
&+ \frac{\Gamma(1+\alpha)}{\Gamma(2 \beta)}\left[\int_{t}^{x} \frac{\left[(x-\xi) A_{1}(\xi)\right]_{\xi}^{\prime} d \xi}{(\xi-t)^{1-2 \beta}}+\int_{0}^{t} \frac{\left[(x-\xi) B_{1}(\xi)\right]_{\xi}^{\prime} d \xi}{(t-\xi)^{1-2 \beta}}\right]+ \\
&+\Gamma(1+\alpha) \xi(x-1) c_{1}(\xi)
\end{aligned}
$$

$$
\begin{aligned}
K_{2}(x, t)=- & \frac{\Gamma(1+\alpha)}{\Gamma(2 \beta)} x\left[\int_{t}^{1} \frac{\left[(1-\xi) A_{1}(\xi)\right]^{\prime} d \xi}{(\xi-t)^{1-2 \beta}}-\right. \\
& \left.-\int_{0}^{x} \frac{\left[(1-\xi) B_{1}(\xi)\right]^{\prime} d \xi}{(t-\xi)^{1-2 \beta}}-\int_{x}^{1} \frac{\left[(1-\xi) B_{1}(\xi)\right]^{\prime} d \xi}{(t-\xi)^{1-2 \beta}}\right]+ \\
& +\frac{\Gamma(1+\alpha)}{\Gamma(2 \beta)} \int_{0}^{x} \frac{\left[(x-\xi) B_{1}(\xi)\right]_{\xi}^{\prime} d \xi}{(t-\xi)^{1-2 \beta}}+\Gamma(1+\alpha) x(1-\xi) c_{1}(\xi) ; \\
f(x)= & \frac{\Gamma(\beta) \Gamma(1+\alpha)}{\Gamma(2 \beta)}\left[x \int_{0}^{1} \frac{(1-\xi) \gamma(\xi)}{\mu(\xi)} d \xi-\int_{0}^{x} \frac{(x-\xi) \gamma(\xi)}{\mu(\xi)} d \xi\right]
\end{aligned}
$$

В силу сделанных предположений относительно гладкости известных функций можно заключить, что

$$
K(x, t) \in C(\bar{I} \times \bar{I}) \cap C^{3}(I \times I), \quad f(x) \in C^{1}(\bar{I}) \cap C^{3}(I) .
$$

Таким образом, уравнение (22) есть уравнение Фредгольма второго рода относительно $\tau(x)$, безусловная разрешимость которого в требуемом классе функций следует из единственности решения задачи.

При выполнении условий (11)-(15) теоремы 1 , используя функциональные соотношения (19) и (21), существование решения задачи (1)-(5) также сведем к разрешимости интегрального уравнения Фредгольма второго рода.

Пусть теперь $\lambda=(1-2 m) / 2, \beta=0$.

Исключив $\nu(x)$ из соотношений $(20)$ и $(21)$, после несложных вычислений придём к уравнению

$$
\tau(x)+\int_{0}^{1} K_{3}(x, \xi) \tau(\xi) d \xi=F(x),
$$


где

$$
\begin{gathered}
K_{3}(x, \xi)= \begin{cases}K_{4}(x, \xi), & \xi \leqslant x \\
K_{5}(x, \xi), & \xi \geqslant x\end{cases} \\
K_{4}(x, \xi)=\Gamma(1+\alpha)\left[(x-1)\left(A_{2}(\xi)+\xi A_{2}^{\prime}(\xi)\right)+\xi(1-x) B_{2}(\xi)\right], \\
K_{5}(x, \xi)=\Gamma(1+\alpha) x\left[(1-\xi)\left(A_{2}^{\prime}(\xi)+B_{2}(\xi)\right)-A_{2}(\xi)\right] \\
F(x)=\Gamma(1+\alpha)\left[\int_{0}^{x}(x-\xi) \gamma_{2}(\xi) d \xi-x \int_{0}^{1}(1-\xi) \gamma_{2}(\xi) d \xi\right]
\end{gathered}
$$

В силу сделанных ранее предположений относительно гладкости известных функций можно заключить, что $K_{3}(x, \xi) \in C(\bar{I} \times \bar{I}) \cap C^{3}(I \times I), F(x) \in$ $C^{1}(\bar{I}) \cap C^{3}(I)$.

Следовательно, уравнение (23) есть уравнение Фредгольма второго рода относительно $\tau(x)$, безусловная разрешимость которого в требуемом классе функций следует из единственности решения задачи (1)-(5).

\section{ORCID}

Олег Александрович Репин: http://orcid.org/0000-0003-1522-3955

Анна Валерьевна Тарасенко: http://orcid.org/0000-0002-0487-8262

\section{БИБЛИОГРАФИЧЕСКИЙ СПИСОК}

1. Самко С. Г., Килбас А. А., Маричев О. И. Интеграль и производные дробного порядка и некоторые их приложения. Минск: Наука и техника, 1987. 688 с.

2. Saigo M. A remark on integral operators involving the Gauss hypergeometric function // Math. Rep. Coll. Gen. Educ., Kyushu Univ., 1978. vol.11, no. 2. pp. 135-143.

3. Репин О. А. Краевые задачи со смещением для уравнений гиперболического и смешанного типов. Саратов, 1992. $164 \mathrm{c}$.

4. Кочубей А. Н. Диффузия дробного порядка // Диффер. уравн., 1990. Т. 26, № 4. С. 660670.

5. Бицадзе А. Н. Некоторые классы уравнений в частных производных. М.: Наука, 1981. $448 \mathrm{c.}$

6. Геккиева С. Х. Аналог задачи Трикоми для уравнения смешанного типа с дробной производной // Известия КБНЦ РАН, 2001. №2(7). С. 78-80.

7. Килбас А. А., Репин О. А. О разрешимости краевой задачи для уравнения смешанного типа с частной дробной производной Римана-Лиувилля // Диффер. уравн., 2010. Т. 46, № 10. С. $1453-1460$.

8. Репин О. А., Кумыкова С. К. Нелокальная задача с дробными производными для уравнения смешанного типа // Изв. вузов. Матем., 2014. № 8. С. 79-85.

9. Репин О. А., Кумыкова С. К. Об одной краевой задаче со смещением для уравнения смешанного типа в неограниченной области // Диффер. уравн., 2012. Т. 48, № 8. С. 11401149.

10. Репин О. А., Кумыкова С. К. Нелокальная задача для уравнения смешанного типа, порядок которого вырождается вдоль линии изменения типа// Изв. вузов. Матем., 2013. № 8 . С. 57-65.

Поступила в редакцию 05/XI/2014;

в окончательном варианте - 11/I/2015;

принята в печать $-25 / \mathrm{II} / 2015$. 
Vestn. Samar. Gos. Techn. Un-ta. Ser. Fiz.-mat. nauki

[J. Samara State Tech. Univ., Ser. Phys. \& Math. Sci.], 2015, vol. 19, no. 1, pp. 78-86

ISSN: 2310-7081 (online), 1991-8615 (print)

doi: http://dx.doi.org/10.14498/vsgtu1398

MSC: 35M12

\title{
NONLOCAL PROBLEM FOR PARTIAL DIFFERENTIAL EQUATIONS OF FRACTIONAL ORDER
}

\author{
O. A. Repin ${ }^{1,2}$, A. V. Tarasenko ${ }^{3}$ \\ 1 Samara State Economic University, \\ 141, Sovetskoy Armii st., Samara, 443090, Russian Federation. \\ 2 Samara State Technical University, \\ 244, Molodogvardeyskaya st., Samara, 443100, Russian Federation. \\ 3 Samara State University of Architecture and Civil Engineering, \\ 194, Molodogvardeyskaya st., Samara, 443001, Russian Federation.
}

\begin{abstract}
A nonlocal problem is investigated for the partial differential equation (diffusion equation of fractional order) in a finite domain. The boundary condition contains a linear combination of generalized operators of fractional integrodifferentiation used on the solution in the characteristics and the solution and its derivative in the degenerating line. The uniqueness of the solution is proved by a modified Tricomi method. The existence of the solution is equivalently reduced to the question of the solvability of Fredholm integral equations of the second kind.
\end{abstract}

Keywords: operator of fractional integro-differentiation, boundary value problem, Fredholm integral equation of the second kind.

doi: http://dx.doi.org/10.14498/vsgtu1398

\section{ORCID}

Oleg A. Repin: http: //orcid.org/0000-0003-1522-3955

Anna V. Tarasenko: http://orcid.org/0000-0002-0487-8262

\section{REFERENCES}

1. Samko St. G., Kilbas A. A., Marichev O. I. Fractional integrals and derivatives: theory and applications. New York, NY, Gordon and Breach, 1993, xxxvi+976 pp.

2. Saigo M. A remark on integral operators involving the Gauss hypergeometric function, Math. Rep. Coll. Gen. Educ., Kyushu Univ., 1978, vol.11, no. 2, pp. 135-143.

(C) 2015 Samara State Technical University.

\section{How to cite Reference}

Repin O. A., Tarasenko A. V. Nonlocal problem for partial differential equations of fractional order, Vestn. Samar. Gos. Tekhn. Univ., Ser. Fiz.-Mat. Nauki [J. Samara State Tech. Univ., Ser. Phys. \& Math. Sci.], 2015, vol. 19, no. 1, pp. 78-86. doi: 10.14498/vsgtu1398. (In Russian)

\section{Authors Details}

Oleg A. Repin (Dr. Phys. \& Math. Sci., Professor; matstat@mail.ru; Corresponding Author), Head of Department, Dept. of Mathematical Statistics and Econometrics ${ }^{1}$; Professor, Dept. of Applied Mathematics \& Computer Science ${ }^{2}$.

Anna V. Tarasenko (Cand. Phys. \& Math. Sci.; tarasenko.a.v@mail.ru), Associate Professor, Dept. of Higher Mathematics. 
3. Repin O. A. Kraevye zadachi so smeshcheniem dlia uravnenii giperbolicheskogo $i$ smeshannogo tipov [Boundary value problems with shift for equations of hyperbolic and mixed type]. Saratov, 1992, 164 pp. (In Russian)

4. Kochubei A. N. Fractional-order diffusion, Differ. Equ., 1990, vol. 26, no. 4, pp. 485-492.

5. Bitsadze A. N. Nekotorye klassy uravnenii v chastnykh proizvodnykh [Some Classes of Partial Differential Equations]. Moscow, Nauka, 1981, 448 pp. (In Russian)

6. Gekkieva S. Kh. An analog of the Tricomi problem for a mixed type equation with a partial fractional derivative, Izvestiya Kabardino-Balkarskaya Nauchnoogo Tsentra RAN, 2001, no. 2(7), pp. 78-80 (In Russian).

7. Kilbas A. A., Repin O. A. Solvability of a boundary value problem for a mixed-type equation with a partial Riemann-Liouville fractional derivative, Differ. Equ., 2010, vol. 46, no. 10, pp. 1457-1464. doi: 10.1134/S0012266110100095.

8. Repin O. A., Kumykova S. K. A nonlocal problem with fractional derivatives for the mixed type equation, Russian Math. (Iz. VUZ), 2014, vol.58, no.8, pp. 65-70. doi: 10.3103/ S1066369X14080088.

9. Repin O. A., Kumykova S. K. On a boundary value problem with shift for an equation of mixed type in an unbounded domain, Differ. Equ., 2012, vol.48, no. 8, pp. 1127-1136. doi : 10.1134/S0012266112080083.

10. Repin O. A., Kumykova S. K. A nonlocal problem for a mixed-type equation whose order degenerates along the line of change of type, Russian Math. (Iz. VUZ), 2013, vol. 57, no. 8, pp. 49-56. doi: 10.3103/S1066369X13080069.

Received 05/XI/2014;

received in revised form $11 / \mathrm{I} / 2015$;

accepted 25/II/2015. 